\title{
Endoparasites infecting exotic captive amphibian pet and zoo animals (Anura, Caudata) in Germany
}

\author{
Malek J. Hallinger ${ }^{1,2}$ (D) Anja Taubert ${ }^{1} \cdot$ Carlos Hermosilla $^{1}$ \\ Received: 30 January 2020 / Accepted: 6 September 2020 / Published online: 22 September 2020 \\ (C) The Author(s) 2020
}

\begin{abstract}
Alongside exotic reptiles, amphibians, such as toads, frogs, salamanders, and newts, are nowadays considered popular pets worldwide. As reported for other exotic pet animals, amphibians are known to harbor numerous gastrointestinal parasites. Nonetheless, very little data are available on captive amphibian parasitic diseases. In this study, we applied direct saline fecal smears (DSFS) to examine in total 161 stool samples from 41 different amphibian species belonging to the orders Anura and Caudata. In addition, carbolfuchsin-smear (CFS) staining ( $n=74$ samples) was used to detect amphibian Cryptosporidium oocysts. Also, complete dissections of deceased amphibians $(n=107)$ were performed to specify parasite infections and to address parasiteassociated pathogenicity. Overall, examined amphibian fecal samples contained 12 different parasite taxa. The order Rhabditida with the species Rhabdias spp. and Strongyloides spp. were the most prevalent nematode species (19.3\%), followed by flagellated protozoans (8.7\%), Amphibiocapillaria spp./Neocapillaria spp. (7.5\%), Oswaldocruzia spp. (4.3\%), Blastocystis spp. (3.1\%), Cosmocerca spp. (3.1\%), oxyurids (Pharyngonoidae) (3.1\%), spirurids (1.2\%), un-sporulated coccidian oocysts $(0.6 \%)$, Tritrichomonas spp. (0.6\%), Karotomorpha spp. (0.6\%), and Cryptosporidium spp. (0.6\%). One CFS-stained fecal sample (1.4\%) was positive for Cryptosporidium oocysts. Within dissected amphibians, $31(48.4 \%)$ of the anurans and $11(26.2 \%)$ of the salamanders were infected with gastrointestinal parasites. One cutaneous Pseudocapillaroides xenopi infection was diagnosed in an adult African clawed frog (Xenopus laevis). Etiologically, 17 (15.9\%) of them died due to severe parasitic and/or bacterial infections (e.g., Chryseobacterium indologenes, Citrobacter freudii, Sphingobacterium multivorum, Klebsiella pneumoniae). High prevalence and pathological findings of several clinical amphibian parasitoses call for more detailed investigation on gastrointestinal parasite-derived molecular mechanisms associated with detrimental lesions or even death.
\end{abstract}

Keywords Amphibians $\cdot$ Parasites $\cdot$ Caudata $\cdot$ Anura $\cdot$ Endoparasites $\cdot$ Exotic pets $\cdot$ Exotic pet medicine

\section{Introduction}

Amphibian species are ectothermic, tetrapod, and mainly carnivorous vertebrate species of the class Amphibia (Hill et al. 2015). Modern amphibians inhabit a wide variety of habitats, with most species living within terrestrial, fossorial, arboreal, or freshwater aquatic ecosystems. In the last decades,

Section Editor: Elizabeth Marie Warburton

Malek J. Hallinger

malekhallinger@web.de

1 Institute of Parasitology, Justus Liebig University Giessen, Schubertstr. 81, Biomedical Research Centre Seltersberg, 35392 Giessen, Germany

2 exomed GmbH, Schönhauser Str. 62, 13127 Berlin, Germany amphibians established themselves as domestic exotic pets/ zoo animals worldwide (Mutschmann 2010). Amphibian species have suffered a significant decline in the wild during the last decades, mainly due to anthropogenic pressure, such as environmental contamination, UV-B irradiation, introduction of alien/invasive species, direct mistreatment, habitat losses, climate changes, and emerging diseases (e.g., chytridiomycosis, Ranavirus) (Daszak et al. 1999; Stuart et al. 2004; Beebee and Griffiths 2005; Collins 2010; Bishop et al. 2012; Henle et al. 2012; Foden et al. 2013; Martel et al. 2014; Wren et al. 2015; Nguyen et al. 2017) and have attracted special media and public attention. This public attention among other factors has raised interest for these ectothermic animals resulting in an increased popularity of amphibians as private pets, also in Germany (Krautwald-Junghanns 2017).

International amphibian trade is becoming governmentally more and more restricted in many countries. Radical 
restrictions seem to be impossible to implement and also require time, effort, and knowledge with no guarantee of success. Even non-regulated trade/black market activities happen as suggested elsewhere (Garner et al. 2009; Bishop et al. 2012). More importantly, diverse anuran (i.e., frogs and toads) and caudate (i.e., salamanders and newts) amphibians threatened by extinction are nowadays part of many zoological collections worldwide (Bishop et al. 2012; Ziegler 2016; Ziegler and Rauhaus 2019).

Since there is a considerable overlap within described diseases for captive amphibians/reptiles/fishes and free-ranging amphibians, caretakers must be mindful on endoparasites and diseases while co-housing amphibians with wild ones or other zoological taxa, such as fishes (Densmore and Green 2007). Parasitic diseases of amphibians are closely related to parasitoses affecting other ectothermic vertebrates (Densmore and Green 2007). Therefore, parasite species can be transmitted from other ectothermic vertebrates to amphibians (i.e., fish ectoparasites, such as the protozoan species, Trichodina spp. and Ichthyobodo spp.) (Densmore and Green 2007; Mutschmann 2010). Most amphibian pets or other lower vertebrates, such as reptiles maintained in captivity, are often associated to inadequate husbandry and mismanagement conditions (Beck and Pantchev 2013; Wolf et al. 2014). In addition, specific intrinsic associated risk factors (e.g., age, sex, species, host immune status) and extrinsic risk factors (e.g., poor hygiene housing conditions, temperature, humidity, animal density, nutrition) might lead to relevant parasitic burdens (Mutschmann 2010; Beck and Pantchev 2013; Hallinger et al. 2019; 2020). Reinfection with resistant reproductive stages of certain endoparasites (e.g., oxyurid eggs) can lead to heavy parasitism and/ or even death of pet reptiles/amphibians (Frank 1981; Pasmans 2008; Beck and Pantchev 2013; Wolf et al. 2014; Hallinger et al. 2018).

Amphibian hosts can be infected with different gastrointestinal parasites, such as protozoans, nematodes, cestodes, trematodes, acanthocephalans, and pentastomids (Frank 1984; Vaucher 1990; Al-Sorakhy and Amr 2003; Barton and Riley 2004; Densmore and Green 2007; Mutschmann 2010). Some of them bear zoonotic potential, such as helminths (Spirometra spp., Gnathostoma spp., Diphyllobothrium spp., Alaria spp., and Echinostoma spp.) and pentastomids (Pentastoma), since most of them are food-borne diseases (Graczyk und Fried 1998; de Górgolas et al. 2003; Dorny et al. 2009; Pantchev und Tappe 2011; Warwick et al. 2012). Up to date, most scientific research have focused on free-ranging amphibians or laboratory animals (Coggins and Sajdak 1982; Cunningham et al. 1996; Hamann et al. 2012; Kuzmin et al. 2003; Loras et al. 2010; Mohammad et al. 2010; Rizvi and Bhutia 2010; Amin et al. 2012; Yildirimhan et al. 2012), but seldom on captivity kept anuran and caudate amphibians. Thus, this comprehensive investigation on German captive amphibian pets of private households and German zoological collections aims to provide current data on the occurrence of gastrointestinal endoparasites, to assess differences in parasite occurrence between privately kept animals and zoo animals considering host species, keeping facility, sex, and/or order/taxon. In addition, we aim to assess presence of zoonotic parasites circulating in amphibian pets and further to gain a better understanding of parasite-derived pathogenicity in these exotic herpetic pets.

\section{Materials and methods}

\section{Fecal samples}

Examined fecal samples originated either from animals owned privately, submitted by owners attending veterinarians, or by different German zoos entities, which had been referred to exomed® laboratory in Berlin, Germany. In order to identify both protozoan and helminth stages, we performed direct saline fecal smears (DSFS) for general parasitological diagnosis according to Barnard and Upton (1994). Clients were also asked to provide a printed form containing individual animal's signalement (i.e., species, sex, age), husbandry circumstances (i.e., origin, animal density, time in owner's possession), previous parasitological examinations, and anthelminthic treatments. At exomed ${ }^{\circledR}$ laboratory, all stool samples were labeled with corresponding forms and reference numbers and finally conserved at $4{ }^{\circ} \mathrm{C}$ for up to $2 \mathrm{~h}$ in a lab refrigerator until further parasitological examination.

For DSFS, a uniform solution was created by mixing $1 \mathrm{~g}$ of amphibian feces at a ratio of $1: 1$ with $0.9 \%$ saline solution, carefully placed on glass cover slides (Nunc) with pipette (Nunc) and finally covered with cover slips $(22 \times 22 \mathrm{~mm}$; Nunc). Both a $100 \times$ and/or $400 \times$ magnification for light microscopy examination (Axio Imager M1®, Zeiss, Jena) equipped with a digital camera were used here.

Consistent, metazoan parasitic stages (i.e., eggs, proglottids, larvae, nematodes) and protozoan parasitic stages (i.e., trophozoites, cysts and oocyst) were identified based on previous morphological/-metric descriptions as reported elsewhere (Frank 1984, 1985; Mutschmann 2010). Samples were classified as "positive" when at least one stage of an endoparasite was found in fecal smears (Table 1). Samples containing apathogenic flagellates/ciliates (e.g., Nyctotherus) or opalozoans (e.g., Opalina spp., Protoopalina spp.) were classified as "negative" according to previous reports (Corliss 1955; Frank 1984, 1985; Densmore and Green 2007; Mutschmann 2010). Additionally, samples were analyzed by carbolfuchsin-stained (CFS) smears for detection of Cryptosporidium oocysts. 
Table 1 Examined fecal samples of amphibians and origin of sender (total $n=161$ ) regarding infection rate with endoparasites $(\%)$

\begin{tabular}{llccc}
\hline $\begin{array}{l}\text { Amphibian order (number of } \\
\text { different examined species) }\end{array}$ & $\begin{array}{l}\text { Common } \\
\text { name }\end{array}$ & No. examined & Origin (private/vet/zoo) & $\begin{array}{l}\text { Positive for } \\
\text { endoparasites (\%) }\end{array}$ \\
\hline Anura (37) & Frogs/toads & 127 & $106 / 13 / 8$ & $58(45.7)$ \\
Caudata (6) & Salamanders & 32 & $24 / 6 / 2$ & $6(9.4)$ \\
unknown (2) & Unknown & 2 & $2 / 0 / 0$ & $0(0.0)$ \\
\hline
\end{tabular}

\section{Amphibian autopsies}

Amphibian corpses of deceased pet animals were necropsied at exomed $\circledast$ laboratory (Table 2, Online supplement). Clients were also asked to provide a printed form containing individual animal's signalement (i.e., species, sex, age) and husbandry circumstances (i.e., origin, animal density, time in owner's possession). Additionally, amphibian hosts were morphologically identified using corresponding published literature (Hofrichter 2000). In addition, pathohistological examinations were performed using standard hematoxylin and eosin (H\&E) batch staining (Buesa 2007) of the following amphibian organs: liver, lungs, intestine, and kidneys. First, a visual inspection of the whole digestive tract was conducted to unveil presence of macroscopic helminth endoparasites. Afterwards, intestinal contents were examined by DSFS method. Morphological identification of endoparasites was performed under a light microscope equipped with a digital camera (Axio Vision M1®, Zeiss, Jena). External examinations and necropsies were performed as described previously by Mutschmann (2010).

\section{Microbiology}

If requested, feces or coelom swabs were inoculated on different agar plates for bacterial and fungal cultivation. As such, further pathogen isolation on sheep blood agars (5\%), MacConkey agars, alongside Sabourand dextrose agars (SDA) (BioMerieux, Charbonnier les Bains, France) was performed. Bacterial isolates were diagnosed by Gram straining, oxidase and catalase tests, as well as a commercially available API 20E/NE® kit (BioMerieux, Charbonnier les Bains, France) as described for poikilothermal vertebrates, such as amphibians and reptiles (Marenzoni et al. 2015; Hallinger et al. 2018).

\section{Results}

From October 2015 to January 2019, coprological analyses of 161 amphibian fecal samples were performed (Table 3). These scat samples originated from 41 different amphibian species enabling to generate representative prevalence data (Online Supplement). In these samples, we recorded 12 different parasite species (please refer to Table 4). Rhabdias and Strongyloides (Rhabditida) were the most prevalent metazoan parasitic genera (19.3\%). Furthermore, 14 samples (8.7\%) contained flagellated protozoans (Metamonada) and 12 samples Amphibiocapillaria/Neocapillaria spp. (7.5\%), 7 samples Oswaldocruzia spp. (4.3\%), 5 samples Blastocystis spp. (3.1\%), 5 samples Cosmocerca spp. (3.1\%), 4 samples oxyurids (Pharyngonoidae) (2.5\%), two samples spirurids $(1.2 \%)$, and one sample un-sporulated coccidian oocysts $(0.6 \%)$. In addition, Tritrichomonas trophozoites $(0.6 \%)$, Karotomorpha trophozoites ( $0.6 \%$ ), and Cryptosporidium oocysts $(0.6 \%)$ were present in the fecal samples $(n=73)$.

Illustrations of selected parasitic stages and histopathological findings are shown in Figs. 1, 2, 3, and 4. Applying CFS staining (Heine 1982), one Australian green tree frog (Litoria caerulea) out of 73 analyzed samples was positive for Cryptosporidium oocysts (see Fig. 4b). According to taxonomic order, parasite infection rates of anuran and caudata amphibians differed significantly (Chi-square test: $\chi^{2}=7.7$, $\mathrm{df}=1, P=0.01 ; r=0.27 ; 95 \%$ CI [0.07-0.46]), being higher in frogs/toads $(51.12 \%)$ than in salamanders (12.88\%). In addition, Rhabdias/Strongyloides infection rates varied within taxon (Fisher's exact test: $P=0.01 ; r=0.44 ; 95 \%$ CI $[0.03$ $0.72]$ ), as such that caudates were less frequently infected (3.13\%) than anurans $(22.83 \%)$. For other detected parasite species, no significant levels were observed within different amphibian hosts. Furthermore, no significant correlation in parasitic burdens was detected in relation to other analyzed

Table 2 Performed necropsies of amphibians, order, and origin of sender $(n=107)$ regarding infection rate with endoparasites $(\%)$

\begin{tabular}{lllcc}
\hline Amphibian order (number of examined species) & Common name & No. examined & Origin (private/vet/zoo) & Positive for endoparasites (\%) \\
\hline Anura (25) & Frogs/toads & 64 & $25 / 10 / 29$ & $31(48.4)$ \\
Caudata (16) & Salamanders & 42 & $22 / 6 / 14$ & $11(26.2)$ \\
Gymnophiona (1) & Caecillians & 1 & $0 / 0 / 1$ & $0(0.0)$ \\
\hline
\end{tabular}


Table 3 Examined fecal samples of amphibians (total $n=161$ )

\begin{tabular}{|c|c|c|c|}
\hline Order/species & Common name & Author/year of description & Examined fecal samples $(n=161)$ \\
\hline Anura & & & 127 \\
\hline Litoria caerulea & Green tree frog & White, 1790 & 31 \\
\hline Dendrobates tinctorius & Dyeing dart frog & Cuvier, 1797 & 21 \\
\hline Agalychnis callidryas & Red-eyed treefrog & Cope, 1862 & 12 \\
\hline Ceratophrys cranwelli & Chacoan horned frog & Barrio, 1980 & 8 \\
\hline Dendrobates sp. & - & - & 7 \\
\hline Dendrobates auratus & Green-and-black poison dart frog & Girard, 1855 & 3 \\
\hline Oophaga pumilio & Strawberry poison frog & Schmidt, 1857 & 3 \\
\hline Ceratophrys ornata & Argentine horned frog & Bell, 1843 & 2 \\
\hline Pyxicephalus adspersus & African bullfrog & Tschudi, 1838 & 2 \\
\hline Ranitomeya ventrimaculata & Reticulated poison frog & Shreve, 1935 & 2 \\
\hline Epipedobates anthonyi & Anthony's poison arrow frog & Noble, 1921 & 3 \\
\hline Xenopus laevis & African clawed frog & Daudin 1802 & 2 \\
\hline Oophaga histrionica & Harlequin poison frog & Berthold, 1845 & 1 \\
\hline Phyllobates vitatus & Golfodulcean poison frog & Cope, 1893 & 1 \\
\hline Phyllobates bicolor & Black-legged poison frog & Duméril and Bibron, 1841 & 1 \\
\hline Bufo regularis & African common toad & Reuss, 1833 & 1 \\
\hline Ranitomeya imitator & Mimic poison frog & Schulte, 1986 & 1 \\
\hline Agalychnis spurelli & Gliding leaf frog & Boulenger, 1913 & 1 \\
\hline Osornophryne guacamayo & Guacamayo plump toad & Hoogmoed, 1987 & 1 \\
\hline Sycirax wampukrum & - & Bravo, 2009 & 1 \\
\hline Phyllobates terribilis & Golden poison frog & Myers, Daly, and Malkin, 1978 & 4 \\
\hline Adelphobates galactonotus & Splash-backed poison frog & Steindachner, 1864 & 2 \\
\hline Phyllomedusa bicolor & Blue-and-yellow frog & Boddaert, 1772 & 1 \\
\hline Megophrys nasuta & Long-nosed horned frog & Schlegel, 1858 & 1 \\
\hline Anaxyrus debilis & North American green toad & Girard, 1854 & 1 \\
\hline Melanophryniscus klappenbachi & Klappenbach's red-bellied frog & Prigioni and Langone, 2000 & 1 \\
\hline Trachycephalus resinifictrix & Amazon milk frog & Goeldi, 1907 & 2 \\
\hline Bombina microdeladigitora & Guangxi firebelly toad & Tian and $\mathrm{Wu}, 1978$ & 1 \\
\hline Bombina orientalis & Oriental fire-bellied toad & Boulenger, 1890 & 1 \\
\hline Kurixalus bisacculus & Taylor's tree frog & Taylor, 1962 & 1 \\
\hline Rhacophorus nigropalmatus & Wallace's flying frog & Boulenger, 1895 & 1 \\
\hline Kurixalus odontotarsus & Serrate-legged small treefrog & Ye and Fei, 1993 & 1 \\
\hline Gastrotheca riobambae & Andean marsupial tree frog & Fowler, 1913 & 1 \\
\hline Dendrobates leucomelas & Yellow-banded poison dart frog & Steindachner, 1864 & 1 \\
\hline Hylarana nigrovittata & Black-striped frog & Blyth, 1856 & 1 \\
\hline Kaloula pulchra & Banded bullfrog & Gray, 1831 & 1 \\
\hline Unknown & - & - & 2 \\
\hline Caudata & & & 32 \\
\hline Ambystoma mexicanum & Axolotl & Shaw and Nodder, 1798 & 23 \\
\hline Tylototriton spp. & - & - & 5 \\
\hline Tylototriton shanjing & Emperor newt & Nussbaum, Brodie, and Yang, 1995 & 2 \\
\hline Ambystoma dumerilii & Lake Patzcuaro salamander & Dugès, 1870 & 1 \\
\hline Salamandra algira & North African fire salamander & Bedriaga, 1883 & 1 \\
\hline Unknown & & & 2 \\
\hline
\end{tabular}

factors, such as keeping facility (zoo, private household), age, sex, group size, and maintenance conditions. Finally, there were no significant differences of parasitic infection rates when comparing alive from deceased amphibians. 
Table 4 Number and percentage of positive amphibians regarding gastrointestinal endoparasite infections (total $n=161 ; 66$ positive and 95 negative)

\begin{tabular}{|c|c|c|c|}
\hline Kingdom/phylum & Parasite genus/species & $\begin{array}{l}\text { Prevalence/host } \\
\text { order }(\%)\end{array}$ & Host species \\
\hline \multirow[t]{4}{*}{ Metazoa/Nematoda } & \multirow{4}{*}{$\begin{array}{l}\text { Rhabditida (Rhabdias spp./ } \\
\text { Strongyloides spp.) }\end{array}$} & Total: 31/161 (19.3) & \\
\hline & & Anura: 29/127 (22.8) & $\begin{array}{l}\text { Agalychnis callidryas (7), Agalychnis spurelli } \\
\text { (1), Bufo regularis (1), Ceratophrys cranwelli } \\
\text { (1), Dendrobates sp. (1), Dendrobates tinctorius } \\
\text { (4), Epipedobates anthonyi (1), Kaloula pulchra } \\
\text { (1), Kurixalus bisacculus (1), Litoria caerulea (6), } \\
\text { Megophrys nasuta (1), Osornophryne guacamayo } \\
\text { (1), Ranitomeya imitator (1) }\end{array}$ \\
\hline & & Caudata: 1/32 (3.1) & Ambystoma mexicanum, metamorphized (1) \\
\hline & & Unknown: $1 / 2(50.0)$ & Unknown (2) \\
\hline \multirow[t]{3}{*}{ Metazoa/Nematoda } & \multirow{3}{*}{$\begin{array}{l}\text { Amphibiocapillaria spp./ } \\
\text { Neocapillaria } \mathrm{spp} .\end{array}$} & Total: 12/161 (7.5) & \\
\hline & & Anura: 11/127 (8.7) & $\begin{array}{l}\text { Agalychnis callidryas (5), Dendrobates } \\
\text { tinctorius (2), Litoria caerulea (3), } \\
\text { Megophrys nasuta (1) }\end{array}$ \\
\hline & & Caudata: 1/32 (3.1) & Tylototriton shanjing (1) \\
\hline \multirow[t]{2}{*}{ Metazoa/Nematoda } & \multirow[t]{2}{*}{ Oswaldocruzia spp. } & Total: 7/161 (4.3) & \\
\hline & & Anura: 7/127 (5.5) & Agalychnis callidryas (6), Litoria caerulea (1) \\
\hline \multirow[t]{2}{*}{ Metazoa/Nematoda } & \multirow[t]{2}{*}{ Cosmocerca spp. } & Total: 5/161 (3.1) & \\
\hline & & Anura: 5/127 (3.9) & $\begin{array}{l}\text { Ceratophrys cranwelli (1), Litoria caerulea (3), } \\
\text { Oophaga pumilio (1) }\end{array}$ \\
\hline \multirow[t]{3}{*}{ Metazoa/Nematoda } & \multirow[t]{3}{*}{ Oxyurids (Pharyngonoidae) } & Total: 4/161 (2.5) & \\
\hline & & Anura: 3/127 (2.7) & Dendrobates tinctorius (1), Litoria caerulea (2) \\
\hline & & Unknown: 1/2 (50.0) & Unknown: $1 / 2(50.0)$ \\
\hline \multirow[t]{2}{*}{ Metazoa/Nematoda } & \multirow[t]{2}{*}{ Spirurids } & Total: 2/161 (1.2) & \\
\hline & & Anura: 2/127 (1.6) & Phyllobates terribilis (2) \\
\hline \multirow[t]{4}{*}{ Protozoa/Metamonada } & \multirow{4}{*}{$\begin{array}{l}\text { Flagellated protozoans } \\
\quad \text { (unspecified) }\end{array}$} & Total: $14 / 161(8.7)$ & \\
\hline & & Anura: 11/127 (8.7) & $\begin{array}{l}\text { Bombina microdeladigitora }(1), \text { Ceratophrys } \\
\text { cranwelli }(2), \text { Ceratophrys ornata }(1), \\
\text { Dendrobates auratus }(2), \text { Dendrobates tinctorius } \\
\text { (3), Litoria caerulea }(2)\end{array}$ \\
\hline & & Caudata: 2/32 (6.3) & Ambystoma mexicanum (1), Tylototriton shanjing (1) \\
\hline & & Unknown: $1 / 2(50.0)$ & Unknown (1) \\
\hline \multirow[t]{3}{*}{ Protozoa } & \multirow[t]{3}{*}{ Blastocystis spp. } & Total: 5/161 (3.1) & \\
\hline & & Anura: 4/127 (3.1) & $\begin{array}{l}\text { Atelopus wampukrum (1), Dendrobates } \\
\text { sp. (1), Dendrobates tinctorius (1), } \\
\text { unknown (1) }\end{array}$ \\
\hline & & Caudata: 1/32 (3.1) & Tylototriton shanjing (1) \\
\hline \multirow[t]{2}{*}{ Protozoa/Apicomplexa } & \multirow[t]{2}{*}{ Unsporulated coccidian oocyst } & Total: 1/161 (0.6) & \\
\hline & & Unknown: $1 / 2(50.0)$ & Unkown (1) \\
\hline \multirow[t]{2}{*}{ Protozoa/Metamonada } & \multirow[t]{2}{*}{ Tritrichomonas spp. } & Total: 1/161 (0.6) & \\
\hline & & Anura: 1/127 (0.8) & Dendrobates auratus (1) \\
\hline \multirow[t]{2}{*}{ Protozoa/Metamonada } & \multirow[t]{2}{*}{ Karotomorpha spp. } & Total: 1/161 (0.6) & \\
\hline & & Anura: 1/127 (0.8) & Dendrobates auratus (1) \\
\hline \multirow[t]{2}{*}{ Protozoa/Apicomplexa } & \multirow[t]{2}{*}{ Cryptosporidium spp. } & Total: 1/161 (0.6) & \\
\hline & & Anura: 1/127 (0.8) & Agalychnis callidryas (1) \\
\hline
\end{tabular}

In total, 42 dissected amphibians out of 107 (39.3\%) were positive for endoparasite infections (Table 5). Twenty amphibians $(18.7 \%)$ died due to severe protozoan- and metazoan-induced enteritis: Spironucleus, Tritrichomonas, ciliate infections (3.7\%), as well as cosmocercosis (2.8\%), amphibiocapillariosis/neocapillariosis (1.9\%), amebosis $(1.9 \%)$, rhabdiosis $(0.9 \%)$, strongyloidiosis $(0.9 \%)$, rhigonemosis $(0.9 \%)$, aplectanosis $(0.9 \%)$, nematotaeniosis 
$(0.9 \%)$, and mesomycetososis $(0.9 \%)$. Other etiological causes identified for amphibian deaths included bacterial infections [Chryseobacterium indologenes, Citrobacter freudii, Sphingobacterium multivorum, Klebsiella pneumoniae, Aeromonas hydrophila, Pseudomonas fluorescens/P. luteola, Stenotrophomonas maltophila, Elizabethkingia spp., and Serratia spp. (33 animals, 30.8\%), mycobacteriosis (4 animals, 3.7\%), brucellosis (1 animal, 0.9\%) and clamydiosis (one animal, 0.9\%)] and/or fungal infections [chytridiomycosis (19 animals, 17.8\%), Candida spp., Mucor amphibiorum, Cladosporium spp., Basidobolus spp., Saprolegnia spp., and chromomycosis (12 animals, 11.2\%)]. Concerning non-infectious death causes, we identified in a Betsileo Madagascar frog (Mantidactylus betsileanus) a severe egg-related stasis most probably due to reproductive disorders. Moreover, in a Dyeing dart frog (Dendroabtes tinctorius), we found an advanced renal carcinoma, and in an African clawed frog (Xenopus laevis), a cutaneous lymphadenoma. In $14(13.1 \%)$ animals, the final cause of death remained unknown. Besides, one Batrachochytrium dendrobatidis-infected axolotl salamander (Ambystoma mexicanum) was co-infected with Ichthyobodo spp. ectoparasites. An overview on dissected animals, the diagnosed endoparasites and combined microbiological, pathological, and pathohistological findings is provided in Table 6.

\section{Discussion}

Parasite infections in free-ranging amphibians seem to appear obligatory worldwide, and thus, very high prevalences of up to 90\% have previously been described (Coggins and Sajdak 1982; Al-Sorakhy and Amr 2003; Amin et al. 2012). For instance, Rizvi et al. (2011) sampled free-ranging amphibians in an Indian Wildlife Sanctuary (Haryana) and found that endemic common dicroglossid frogs (Euphlyctis cyanophlyctis) were frequently infected $(52.9 \%)$ by nematodes. In contrast to this wildlife study, there is very little knowledge on parasitic infections of dicroglossid frogs (E. cyanophlyctis) kept in captivity. While comparing our prevalence data with previous published studies, it should be considered that most of these surveys were conducted in wild animals, and this fact might explain prevalence differences. Most likely to dicroglossid frogs, other freeranging amphibians are also showing higher parasitic prevalences when compared with those kept in captivity (Coggins and Sajdak 1982; Amin et al. 2012). Moreover, sensitivity and specificity of applied DSFS to detect helminth and protozoan stages might have influenced observed prevalence as different diagnostic methods in former wildlife studies have been used (Rizvi et al. 2011; Amin et al. 2012).

Despite the fact that extrinsic risk factors, such as habitat changes, habitat losses, predatory pressure, and poor water
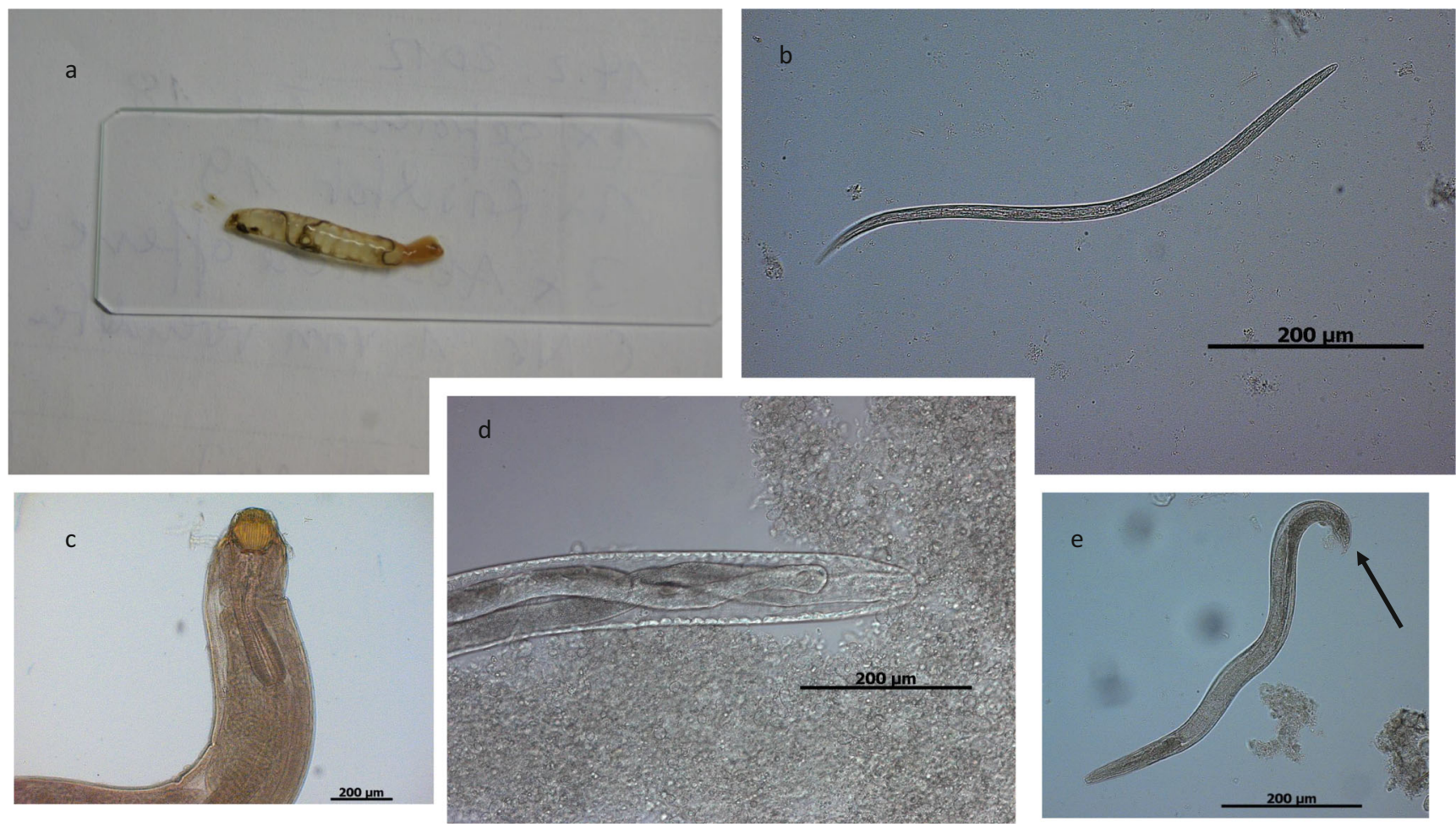

Fig. 1 Selected pictures of helminth endoparasites. a Rhabdias sp.: adult nematodes inside the lung of a red-tailed knobby newt (Tylototriton kweichowensis). b Pseudocapillaria sp.: elongated nematode shed by red-eyed multicolored tree frog (Agalychnis callidryas). c Camallanus sp.: from a Spanish newt, Pleurodeles waltl. Please note the anterior buccal capsule armed with teeth. d Esophagus of Capillaria sp.: shed by an Eastern newt (Notophthalmus viridescens). e Free-living adult male of Rhabdias sp.: please notice posterior end mid gubernaculum, spirules, and cloaca (arrow) 

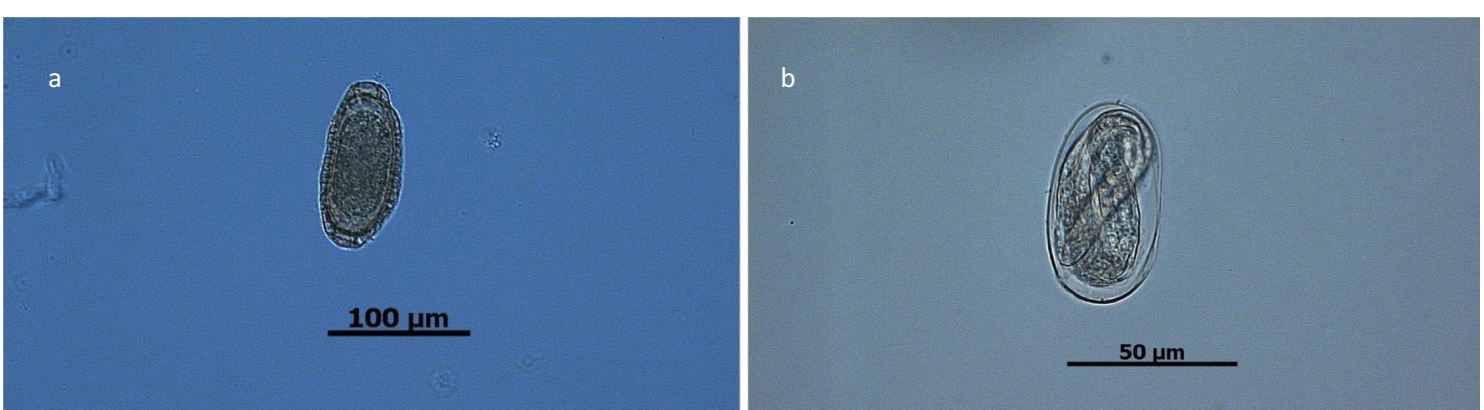

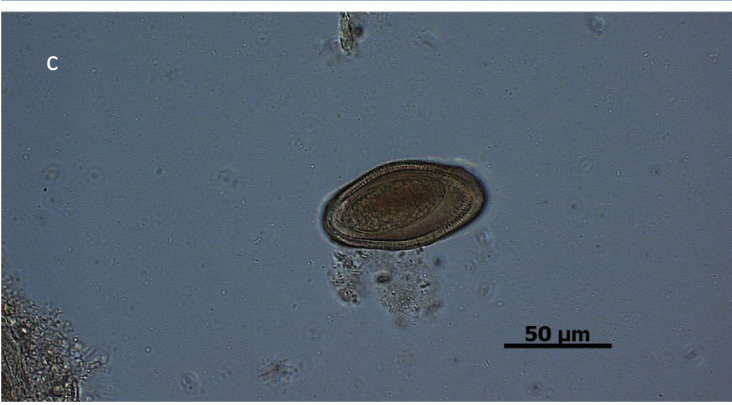

Fig. 2 Selected shed stages of endoparasitic nematodes. a Amphibiocapillaria $\mathrm{sp}$./Neocapillaria $\mathrm{sp}$.: brownish lemon-shaped eggs with two pole-clots. Shed by a crocodile newt (Tylototriton sp.). b Egg of Rhabdias sp.: shed by a Marañón Poison frog (Excidobates mysteriosus). c

quality can directly affect parasitic burdens and prevalences in free-ranging amphibians (Vaucher 1990; Kehr and Hamann 2003; Marcogliese and Pietrock 2011; Thiemann and Wassersug 2000), very little is still known whether these factors might also influence the outcome of parasitic burdens in pet amphibians kept in households or zoos (Mutschmann 2010).

In this study, helminth infections occurred frequently in investigated animals (Table 4). All nematode species found in this survey have been reported to possess pathogenic significance for amphibians (Mutschmann 2010; Amin et al. 2012; Langford and Janovy 2009; Langford 2010; Yildirimhan et al. 2012). Correspondingly, amphibians are

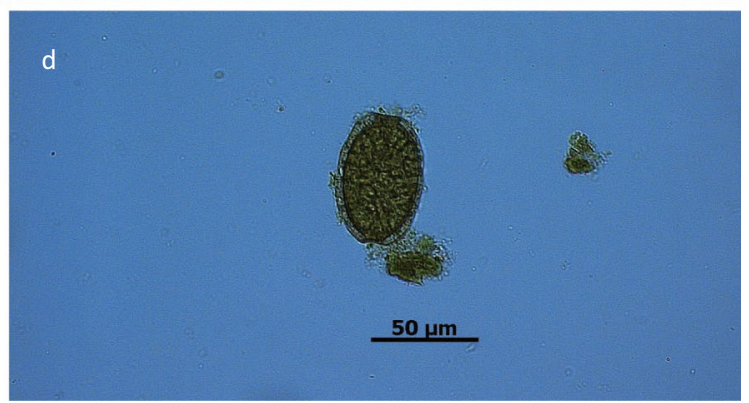

Oxyurid egg: bean-shaped, thick-walled eggs containing a morula. Shed by an Australian green tree frog (Litoria caerulea). d Amphibiocapillaria sp./ Neocapillaria sp.: brownish lemon-shaped eggs with two pole-clots. Shed by a white-lipped horned toad (Megophrys major)

well-known to be parasitized by numerous nematode families, such as Trichinellidae, Rhabditidae, Strongyloididae, Ascarididae, Cosmocercodidae, Oxyuridae, Heterakidae, Camalladae, Gnathostomatidae, Habronematidae, Filaroidae and Physalopteridae. For amphibians, particularly rhabditidean helminths are considered as pathogenic endoparasites (Mutschmann 2010; Amin et al. 2012; Yildirimhan et al. 2012). The genus Strongyloides is known to cause protein-loss enteropathy in various anuran hosts (PattersonKane et al. 2001). Cosmopolitan adult female Rhabdias lungworms are capable of parthenogenesis and known to parasitize lung tissues of different amphibian hosts, including various toad and frog species (Langford 2010; Fernández Loras

Fig. 3 Histology of un-identified nematode infection in a smooth frog (Theloderma licin). a Notice adult nematode inside the intestinal lumen and diverse site-gated mucosal attached stages (arrows). b Enveloped intestinal larval stage: notice thick cuticula of the nematode (arrow)
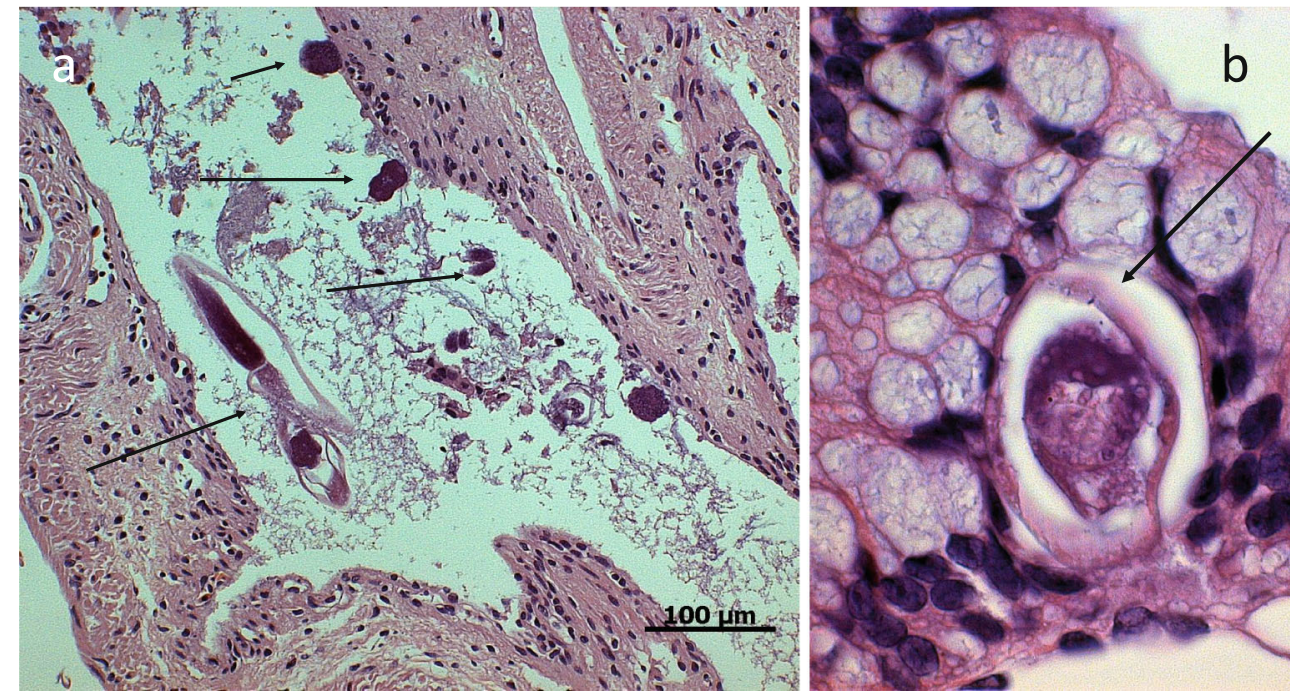
et al. 2011), while males live in earth/ground substrates (geohelminths). Amphibian hosts become infected by oral uptake or percutaneous infection of exogenous infective thirdstage larvae (L3) which then migrate via blood/lymph system into the lungs (Langford and Janovy 2009; Langford 2010). In lungs, adult Rhabdias females start producing eggs through parthenogenesis. Thus, amphibian rhabdiosis might result in pulmonary tissue damage and/or eosinophilic pneumonia (Densmore and Green 2007). In free-ranging amphibians, Rhabdias infections seem to occur frequently and sometimes result in pneumonia (Kuzmin et al. 2003; Mohammad et al. 2010; Fernández Loras et al. 2011). Consistently, Rhabdias spp. infection rates for captive German amphibians were rather high in this study (19.3\%) and resulted in the most prevalent parasites. Rhabdias/Strongyloides infection rates varied significantly within taxon, i.e., caudates were less frequently infected $(3.13 \%)$ than anurans (22.83\%). Nonetheless, it is well known from literature that Rhabdias is more frequently parasitizing frogs/toads (Langford and Janovy 2009; Langford 2010). In line, Rhabdias ranae seems not capable to infect caudates and to be restricted to frogs/toads as suitable hosts, but in the past two decades, first Rhabdias infections in caudates have been reported (Kuzmin et al. 2001; Kuzmin et al. 2003; Eisenberg and Pantchev 2009). Therefore, it seems assumable that anurans might be more often infected with
Rhabdias than caudates, especially because the correlation was rather high $(r=0.44)$ when comparing these two amphibian groups (Cohen 1988). Clinical relevance of rhabdiosis was also underlined in dissections, since in one adult male Australian green tree frog (L. caerulea), a Rhabdias spp.-infected lung was found and which might have caused severe pneumonia, hepatitis, and nephritis. Nonetheless, other pathogens could not be ruled out as the same animal showed secondary bacterial infections with Chryseobacterium indologenes (+++) and Pseudomonas fluorescens (+) isolated from the frog's coeloma. Alongside Rhabdias, other nematode genus, i.e., Oswaldocruzia, was frequently diagnosed $(2.8 \%)$ in domestic kept amphibian pets. Oswaldocruzia nematodes infect amphibian hosts exclusively by the oral uptake of exogenous infective L3 (Hendrikx 1983). Noteworthy, a cutaneous Pseudocapillaroides xenopi infection was diagnosed in an adult African clawed frog $(X$. laevis). This $X$. laevis-infected animal suffered not only of a severe verminous dermatitis but also of secondary Gram-negative P. fluorescens (+++), Aeromonas hydrophila (++), and Citrobacter braakii (++) dermal infections. The amphibian nematode $P$. xenopi infects the epidermis and can cause clinically symptoms, such as erythematous/erosive dermatitis, with characteristic roughness of affected skin, petechiae, and dermal ulcera (Cunningham et al. 1996; Mutschmann 2010).
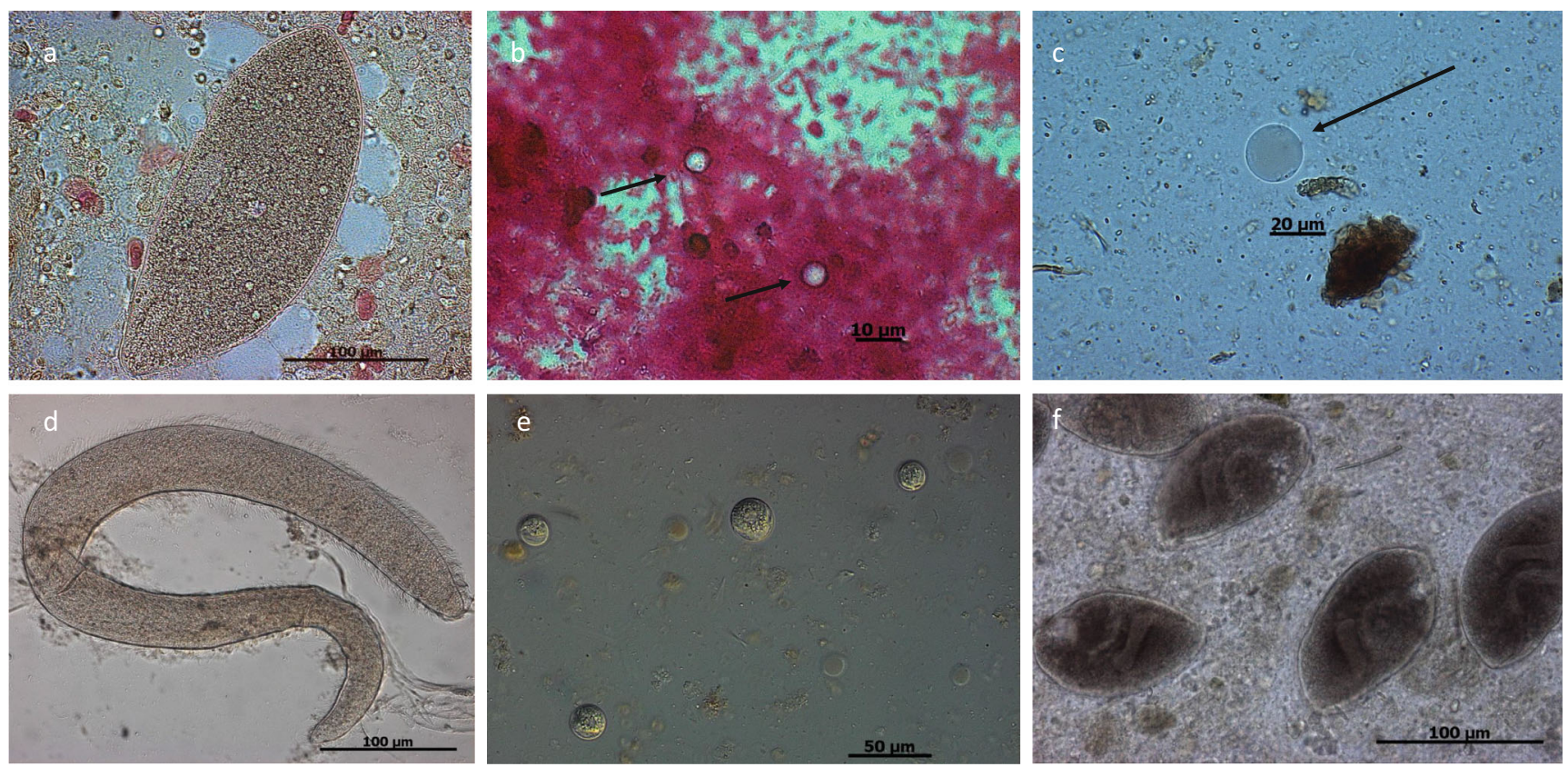

Fig. 4 Selected shed stages of protozoan endoparasites/commensals in amphibians. a Opalina sp.: heterokont from a yellow-bellied toad

Cranwells horned frog (Ceratophrys cranwelli). d Protoopalina sp.: heterokont form Hyperolius sp., Protoopalina sp., such as Opalina sp., seem to be most likely commensal, than parasitic. e Spores of Basidobolus sp.: Basidobolus is a filamentous fungus known to cause zygomycosis in amphibians, and shed spores can easily be mistaken for un-sporulated coccidian oocysts. f Trophozoites of Nyctotherus sp.: large trophozoites with lateral cytostomes and prominent macronuclei. Shed by red-eyed multicolored tree frog (Agalychnis callidryas) 
Table 5 Number and percentage of positive amphibian corpses regarding gastrointestinal endoparasite infections (total $n=107 ; 42$ positive and 14 different gastrointestinal parasites detected)

\begin{tabular}{|c|c|c|c|}
\hline Kingdom/phylum & Parasite species & Prevalence $(\%)$ & Host species $(n)$ \\
\hline \multirow[t]{3}{*}{ Metazoa/Nematoda } & \multirow{3}{*}{$\begin{array}{l}\text { Amphibiocapillaria } \\
\text { spp./Neocapillaria spp. }\end{array}$} & Total: 8/107 (7.5) & \\
\hline & & Anura: 6/64 (9.4) & $\begin{array}{l}\text { Dendrobates tinctorius (1), Hylarana cubitalis } \\
\text { (1), Kurixalus bisacculus (1), Litoria caerulea } \\
\text { (1), Rhinella marina (1), Xenopus laevis (1) }\end{array}$ \\
\hline & & Caudata: 2/42 (4.8) & $\begin{array}{l}\text { Notophthalmus viridescens }(1) \text {, } \\
\text { Triturus pygmaeus }(1)\end{array}$ \\
\hline \multirow[t]{3}{*}{ Metazoa/Nematoda } & \multirow[t]{3}{*}{ Cosmocerca spp. } & Total: 8/107 (7.5) & \\
\hline & & Anura: 5/64 (7.8) & $\begin{array}{l}\text { Bombina bombina (1), Dendrobates sp. (1), } \\
\text { Hylarana cubitalis (1), Litoria caerulea (1), } \\
\text { Mantidactylus betsileanus (1) }\end{array}$ \\
\hline & & Caudata: $3 / 42$ (7.1) & $\begin{array}{l}\text { Salamandra algira splendens }(2), \\
\text { Salamandra salamandra (1) }\end{array}$ \\
\hline \multirow[t]{3}{*}{ Metazoa/Nematoda } & \multirow{3}{*}{$\begin{array}{l}\text { Rhabditida (Rhabdias } \\
\text { spp./Strongyloides spp.) }\end{array}$} & Total: 3/107 (2.8) & \\
\hline & & Anura: 2/64 (3.1) & Dendrobates auratus (1), Litoria caerulea (1) \\
\hline & & Caudata: $1 / 42(2.4)$ & Salamandra crexpoi (1) \\
\hline \multirow[t]{2}{*}{ Metazoa/Nematoda } & \multirow[t]{2}{*}{ Oswaldocruzia spp. } & Total: 3/107 (2.8) & \\
\hline & & Anura: 3/64 (4.7) & Bombina variegata (1), Dendrobates tinctorius (2) \\
\hline \multirow[t]{3}{*}{ Metazoa/Nematoda } & \multirow[t]{3}{*}{ Aplectana spp. } & Total: 3/107 (2.8) & \\
\hline & & Anura: 2/64 (3.1) & Dendrobates auratus (1), Hylarana cubitalis (1) \\
\hline & & Caudata: 1/42 (2.4) & Salamandra salamandra (1) \\
\hline \multirow[t]{2}{*}{ Metazoa/Platyhelminthes } & \multirow[t]{2}{*}{ Cestodes (unspecified) } & Total: 2/107 (2.8) & \\
\hline & & Anura: 3/64 (4.7) & $\begin{array}{l}\text { Bombina variegata (1), Dendrobates tinctorius } \\
\text { (1), Hylarana cubitalis (1) }\end{array}$ \\
\hline \multirow[t]{2}{*}{ Metazoa/Trematodes } & \multirow[t]{2}{*}{ Trematode eggs (Digenea) } & Total: 1/107 (1.4) & \\
\hline & & Anura: 1/64 (1.6) & Bombina variegata $(1)$ \\
\hline \multirow[t]{3}{*}{ Protozoa/Metamonada } & \multirow[t]{3}{*}{ Trichomonas spp. } & & \\
\hline & & Anura: 6/64 (9.3) & $\begin{array}{l}\text { Dendrobates tinctorius (1), Oophaga histrionica } \\
\text { (1), Phyllobates terribilis (1), } \\
\text { Theloderma corticale (3) }\end{array}$ \\
\hline & & Caudata: $1 / 42(0.2)$ & Ambystoma tigrinum (1) \\
\hline \multirow[t]{3}{*}{ Protozoa/Metamonada } & \multirow{3}{*}{$\begin{array}{l}\text { Flagellated protozoa } \\
\text { (unspecified) }\end{array}$} & Total: 5/107 (4.7) & \\
\hline & & Anura: 2/64 (3.1) & Phyllobates bicolor (1), Ranitomeya imitator (1) \\
\hline & & Caudata: 3/42 (7.1) & $\begin{array}{l}\text { Cynops pyrrhogaster (1), } \\
\quad \text { Triturus pygmaeus (1), Tylototriton sp. (1) }\end{array}$ \\
\hline \multirow[t]{3}{*}{ Protozoa/Metamonada } & \multirow[t]{3}{*}{ Spironucleus spp. } & Total: 5/107 (4.7) & \\
\hline & & Anura: 1/64 (1.6) & Gastrotheca riobambae (1) \\
\hline & & Caudata: $4 / 42(9.5)$ & $\begin{array}{l}\text { Ambystoma tigrinum (1), Cynops pyrrhogaster } \\
\text { (1), Salamandra crexpoi (1), } \\
\text { Salamandra salamandra (1) }\end{array}$ \\
\hline \multirow[t]{2}{*}{ Protozoa } & \multirow[t]{2}{*}{ Entamoeba spp. } & Total: 3/107 (2.8) & \\
\hline & & Anura: 3/64 (4.7) & $\begin{array}{l}\text { Atelopus hoogmoedi (1), Litoria caerulea } \\
\quad \text { (1), Oophaga histrionica (1) }\end{array}$ \\
\hline \multirow[t]{2}{*}{ Protozoa } & \multirow[t]{2}{*}{ Neobalantidium spp. } & Total: 3/107 (2.8) & \\
\hline & & Anura: 3/64 (4.7) & Theloderma corticale (1), Xenopus laevis (2) \\
\hline \multirow[t]{2}{*}{ Protozoa/Metamonada } & \multirow[t]{2}{*}{ Tritrichomonas spp. } & $\begin{array}{l}\text { Total: } 3 / 107(2.8) \\
\text { Caudata: } 3 / 42(7.1)\end{array}$ & \\
\hline & & Caudata: 3/42 (7.1) & $\begin{array}{l}\text { Salamandra crexpoi (1), Salamandra } \\
\quad \text { salamandra (1), Tylototriton sp. (1) }\end{array}$ \\
\hline \multirow[t]{2}{*}{ Protozoa/Metamonada } & \multirow[t]{2}{*}{ Karotomorpha spp. } & Total: 3/107 (2.8) & \\
\hline & & Anura: 3/64 (4.7) & $\begin{array}{l}\text { Oophaga histrionica }(1) \\
\text { Theloderma corticale }(2)\end{array}$ \\
\hline
\end{tabular}


Table 5 (continued)

\begin{tabular}{llll}
\hline Kingdom/phylum & Parasite species & Prevalence $(\%)$ & Host species $(n)$ \\
\hline Protozoa & Nyctotherus spp. & Total: $2 / 107(1.9)$ & \\
& & Anura: $2 / 64(3.1)$ & Xenopus laevis (2) \\
Protozoa/Apicomplexa & Eimeria sp. & Total: $1 / 107(0.9)$ & \\
& & Caudata: $1 / 42(0.2)$ & Salamandra salamandra (1) \\
\hline
\end{tabular}

P. xenopi can complete its direct life cycle within epidermis of frogs/toads in which burrowing activities of subdermal nematodes can lead to the damage of parasitized skin. Therefore, $P$. xenopi-infected animals are more susceptible for bacterial and/or fungal secondary dermal infections (Cunningham et al. 1996), as confirmed in our investigation.

According to protozoan enteric infections, in 14 cases (8.7\%), potentially pathogenic, flagellated protozoan genera, such as Proteromonadida, Reteromonadida, Diplomonadida, and Trichomonadida, were additionally diagnosed. Nonetheless, the literature considers many of these enteric flagellates as commensals within intestinal tract of amphibians (Densmore and Green 2007; Mutschmann 2010). Conversely, some genera of diplomonadids (Giardia, Hexamita, Spironucleus) and trichomonadids (Monocercomonas, Hexamastix, Tritrichomonas) can cause weight loss, general edema, and enteritis in severely infected animals.

The clinical relevance of flagellated protozoan infections was demonstrated during conducted dissections: Out of all dissected animals, four $(3.7 \%)$ died because of severe Tritrichomonas spp.- and/or Spironucleus spp.-derived enteritis. These animals showed severe catarrhalic- to hemorrhagicnecrotic enteritis combined with secondary bacterial infections (e.g., Pseudomonas spp./Sphingobacterium spp.) of liver and gut mucosa.

Only five animals $(3.7 \%)$ were positive for Blastocystis spp. infections. Conversely to our findings, Yoshikawa et al. (2004) found anurans and newts from distinct locations in Japan to be infected with Blastocystis showing very high prevalences (47.8-100\%) by using in vitro culture diagnostic methods. Our observed Blastocystis prevalence might have been higher if this in vitro cultivation method would have been applied, but it cannot be excluded that this parasite is simply less frequently found in German pet amphibians. Unfortunately, there is still very little knowledge on amphibian-related blastocystiosis. The same holds true for its possible impact on animal health kept in captivity (Mutschmann 2010). Nevertheless, Blastocystis should be considered as potentially pathogenetic protozoan species and infections should be considered according to clinical symptoms. Moreover, during dissections we here diagnosed Entamoeba spp. cysts in three $(2.8 \%)$ animals.
Several studies have focused on gastrointestinal apicomplexan coccidian parasites in amphibians (Duszynski et al. 2007). So far, monoxenous coccidian genera Eimeria, Goussia, Hyaloklossia, and Cystoisospora (former Isospora according to new nomenclature) have been described in diverse amphibian host species (Duszynski et al. 2007), and for further review a disposed online version (http://biology.unm. edu/coccidia/anura.html) is recommended. In accordance with these reports, we also diagnosed un-sporulated coccidian oocysts in one animal $(0.6 \%)$, but amphibian oocysts were not fully identifiable to species level. Furthermore, non-sporulated Eimeria spp. oocysts were found within gut lumen of one dissected fire salamander, (Salamandra salamandra) but coccidian-derived death was ruled out as this animal was also co-infected with Aplectana spp., Spironucleus spp., and Tritrichomonas sp. and showed a manifested mycotic dermatitis.

Enteropathogenic apicomplexan Cryptosporidium is known to infect also the microvillus border of amphibian gastrointestinal epithelial cells (Jirků et al. 2008). Consistently, we diagnosed Cryptosporidium oocysts in an Australian frog (L. caerulea) via CFS analysis. If here identified Cryptosporidium oocysts were shed during a patent infection or whether they were passed because of Cryptosporidium spp.-infected prey animal consumption (e.g., feeding of baby mice) remains unclear. Since Cryptosporidium can be transmitted by ingestion of infected food animals, poorly treated water as well as direct contact with infective oocysts, it is possible to assume that human infections might occur through ingestion of under-cooked frog (Rana spp.) meat and/or handling and processing of Cryptosporidium-infected frogs as recently demonstrated in Africa (Kia et al. 2017). Former study revealed a high prevalence of Cryptosporidium spp. (35.9\%) in the intestine of 117 frogs (Rana spp.) sold at the Hanwa frog market Zaria, Kaduna State, Nigeria, for human consumption (Kia et al. 2017; Kia and Ukuma 2017). Therefore, further public health studies on different transmission routes of this neglected anthropozoonotic parasite should be conducted, including amphibians designated for human consumption (Kia et al. 2017; Kia and Ukuma 2017).

Aside from protozoans, nematodes, cestodes, and trematodes, no acanthocephalan infections were here detected. Nonetheless, during necropsies, also cestode-parasitized 

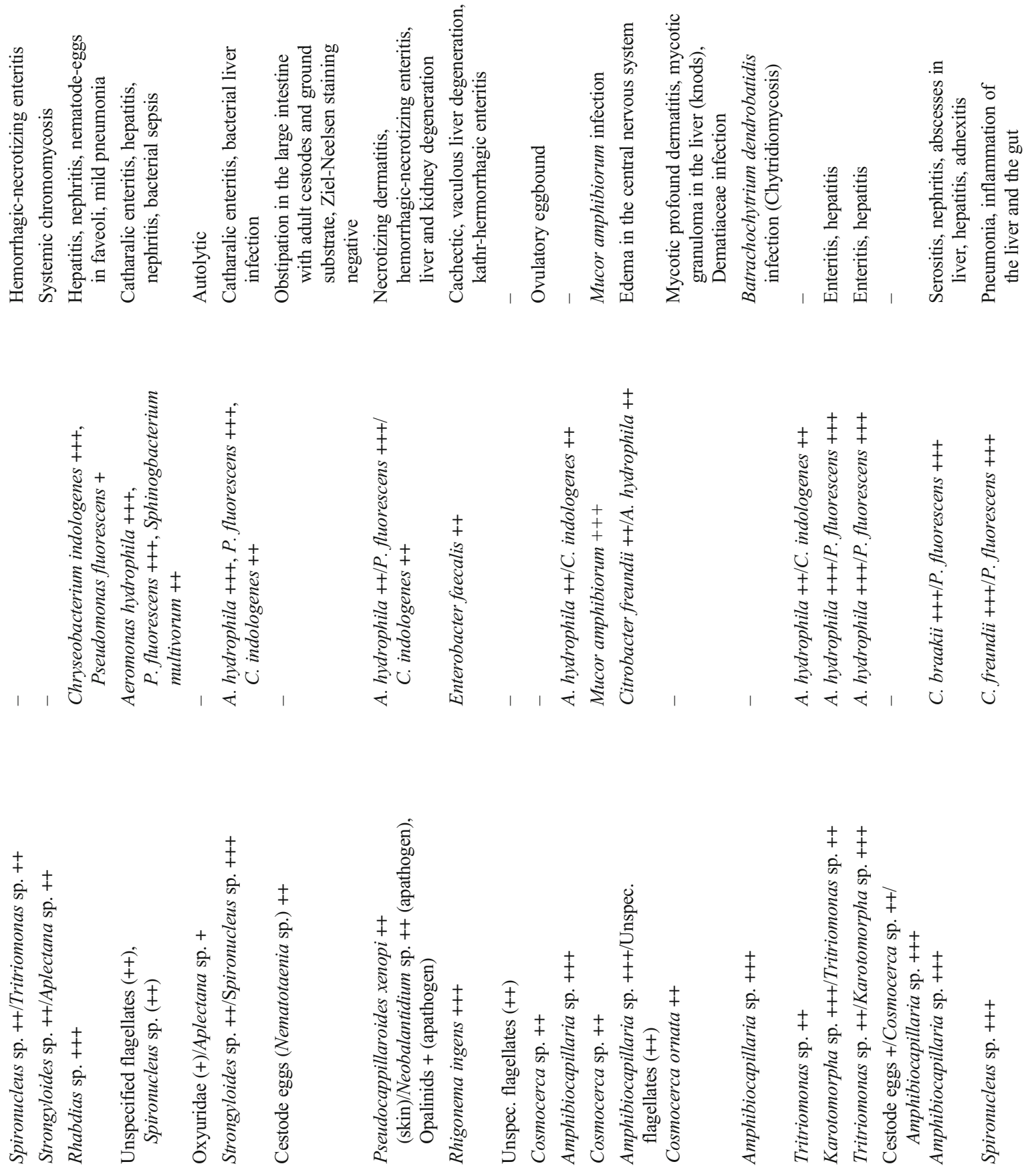

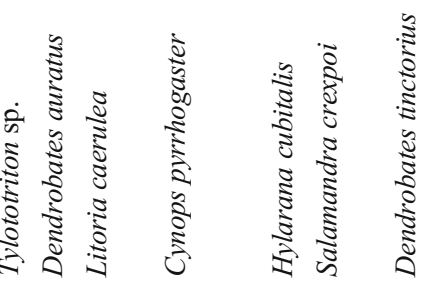
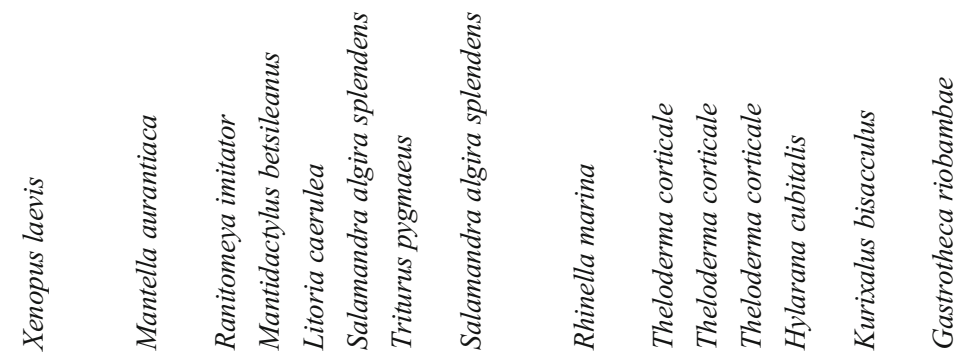


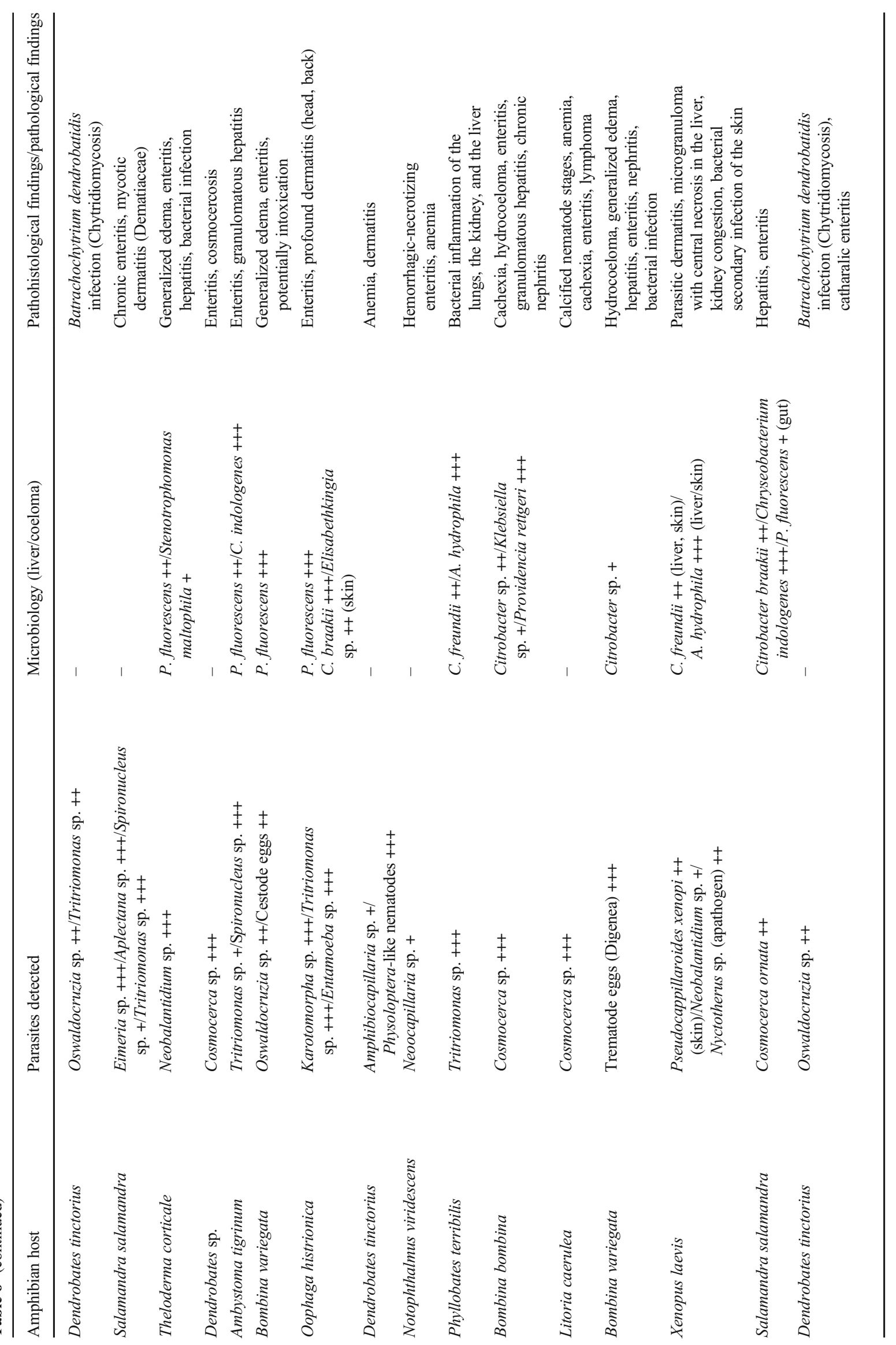


animals were found. As such, in three dissected animals (2.8\%), various long cestode specimens containing mature proglottids were diagnosed. Noteworthy was a heavily Nematotaenia-infected male Dyeing dart frog (Dendrobates tinctorius), which showed obstipation and congestion of ground substrate in the gut lumen. Amphibians are known to be infected by different cestode genera, i.e., Proteocephalus, Ophiotaenia, Cephalochlamys, Bothriocephalus, Nematotaenia, Distoichometra, Cylindrotaenia, and Baerietta. Clinical symptoms of nematotaeniosis manifest in affected animals during stress and/or in case of heavy infections (Mutschmann 2010). Then, ileus with obstipation, blood loss, necrosis of intestinal mucosa, edema or even death may also occur if untreated (Mutschmann 2010). Interestingly, a digenean trematode infection was found in a deceased yellowbellied toad (Bombina variegata), showing clinical symptoms, including hydrocoeloma, generalized edema, and pathohistological findings, such as hepatitis, enteritis, nephritis, and a bacterial co-infection (Citrobacter spp. +). Amphibians represent not only intermediate hosts for various digenean trematode orders (e.g., Amphistomida, Echinostomatida, Gasterostomida, Hemiurida, Holostomida, Plagiorchida) but also second or even final hosts. Nevertheless, trematode-driven pathological effects are mostly unknown for amphibians (Mutschmann 2010).

Since many of examined amphibians in this study are considered as threatened endemic species of neotropical regions, e.g., Adelphobates galactonotus, Phyllomedusa bicolor, and Trachycephalus resinifictrix, and thus being kept as zoo animals for conversation reasons, detected parasites in these animals might represent imported parasites from their natural tropical habitats. Therefore, it seems noteworthy to mention that new wild amphibians introduced into zoological gardens should undergo a mandatory quarantine regime in order to avoid further spread of neozoan parasites as suggested elsewhere (Hallinger et al. 2019; 2020).

Interestingly, parasitic infection rates in investigated anurans $(51.12 \%)$ were significantly higher than the ones observed in caudate species $(12.88 \%)$. As proposed for zoo animals, it is also recommended that newly purchased frogs, newts, and toads by private owners should be submitted to parasitological examination in order to detect presence of gastrointestinal parasites during quarantine as a routine health screening.

\section{Conclusions}

Our representative epidemiological survey on endogenous parasites of captive amphibians in Germany found several pathogenic parasite species resulting in clinically manifested disease. If correctly diagnosed, identified parasitoses should be medicated taking into account commensalism, metabolic features of amphibians, clinical signs, and more importantly prophylactic approaches in order to prevent future infections. Applied DSFS technique on scat samples proved to be valid for detection of many relevant parasitic stages, including tiny protozoan oocysts. Since biology, epidemiology, as well as pathogenesis of most amphibian parasitoses are not well understood, further investigations in these directions are needed. Similarly, the current lack of suitable therapy options for many of these amphibian parasitoses calls for more research in new drug development within the field of neglected herpetology medicine.

Acknowledgements This research was supported by exomed GmbH laboratory in Berlin, Germany, and the Institute of Parasitology of the Justus Liebig University in Giessen, Germany. We would like to thank our colleagues Jaqueline Dahms, Nina Heindorf, Kim Kwauka, Mathias Nolde, and Anna Dieudonne for aiding while working in diagnosis and greatly improving research activities at exomed laboratory. We owe a special debt of gratitude and respect to our colleague Dr. Frank Mutschmann who sadly deceased in November 2018. The article is dedicated to the memory of Dr. Frank Mutschmann and his outstanding work as a passionate veterinarian and parasitologist in the field of herpetology.

Funding Open Access funding enabled and organized by Projekt DEAL.

\section{Compliance with ethical standards}

Conflict of interest The company BioMerieux did neither play a role in the study design nor in collection, analysis, and interpretation of generated data, nor in the decision to submit the manuscript for further publication. None of the authors have any financial or personal relationships that could inappropriately influence or bias the content of the manuscript.

Open Access This article is licensed under a Creative Commons Attribution 4.0 International License, which permits use, sharing, adaptation, distribution and reproduction in any medium or format, as long as you give appropriate credit to the original author(s) and the source, provide a link to the Creative Commons licence, and indicate if changes were made. The images or other third party material in this article are included in the article's Creative Commons licence, unless indicated otherwise in a credit line to the material. If material is not included in the article's Creative Commons licence and your intended use is not permitted by statutory regulation or exceeds the permitted use, you will need to obtain permission directly from the copyright holder. To view a copy of this licence, visit http://creativecommons.org/licenses/by/4.0/.

\section{References}

Al-Sorakhy MK, Amr ZS (2003) Platyhelminth parasites of some amphibians in Jordan. Turk J Zool 27(2):89-93

Amin OM, Düșen S, Oğuz MC (2012) Review of the helminth parasites of Turkish anurans (Amphibia). Sci Parasitol 13(1):1-16

Barton DP, Riley J (2004) Raillietiella indica (Pentastomida) from the lungs of the giant toad, Bufo marinus (Amphibia), in Hawaii, U.S.A. Comp Parasitol 71:251-254. https://doi.org/10.1654/4134

Barnard SM, Upton SJ (1994) A veterinary guide to the parasites of reptiles Vol I Protozoa. Krieger Publishing Company, Malabar, pp $1-98$ 
Beebee TJC, Griffiths RA (2005) The amphibian decline crisis: a watershed for conservation biology? Biol Conserv 125:271-285. https:// doi.org/10.1016/j.biocon.2005.04.009

Beck W, Pantchev N (2013) Practical parasitology in pets. Practical parasitology in pets., (Ed. 2). https://www.cabdirect.org/cabdirect/ abstract/20133176420

Bishop PJ, Angulo A, Lewis JP, Moore RD, Rabb GB, Moreno JG (2012) The amphibian extinction crisis - what will it take to put the action into the amphibian conservation action plan?. SAPI EN S Surveys and Perspectives Integrating Environment and Society, (5.2)

Buesa RJ (2007) Histology: a unique area of the medical laboratory. Ann Diagn Pathol 11(2):137-141. https://doi.org/10.1016/j.anndiagpath. 2007.01.002

Coggins JR, Sajdak RA (1982) A survey of helminth parasites in the salamanders of certain anurans from Wisconsin. Proc Helminthol Soc Wash 49(1):99-102

Cohen J (1988) Statistical power analysis for the behavioral sciences, 2nd edn. Lawrence Erlbaum Associates, New Jersey

Collins JP (2010) Amphibian decline and extinction: what we know and what we need to learn. Dis Aquat Org 92:93-99. https://doi.org/10. 3354/dao02307

Corliss JO (1955) The Opalinid Infusorians: flagellates or ciliates? J Protozool 2(107):114-114. https://doi.org/10.1111/j.1550-7408. 1955.tb02410.x

Cunningham AA, Sainsbury AW, Cooper JE (1996) Diagnosis and treatment of a parasitic dermatitis in a laboratory colony of African clawed frogs (Xenopus laevis). Vet Rec 138:640-642. https://doi. org/10.1136/vr.138.26.640

Daszak P, Berger L, Cunningham AA, Hayatt AD, Green DE, Speare R (1999) Emerging infectious diseases and amphibian population declines. Emerg Infect Dis 5:735-748. https://doi.org/10.3201/ eid0506.990601

de Górgolas M, Santos-O'Connor F, Gárate T, Guarch Troyas RM, Unzú AL, Grobusch MP, Fernández-Guerrero ML (2003) Cutaneous and medullar gnathostomiasis in travelers to Mexico and Thailand. J Travel Med 10(6):358-361

Densmore CL, Green DE (2007) Diseases of amphibians and reptiles. ILAR J 48:235254. https://doi.org/10.1163/157075407782424502

Dorny P, Praet N, Deckers N, Gabriel S (2009) Emerging food-borne parasites. Vet Parasitol 163:196-206

Duszynski DW, Bolek MG, Upton SJ (2007) Coccidia (Apicomplexa: Eimeriidae) of amphibians of the world. Zootaxa 1667(1):1-77

Eisenberg T, Pantchev N (2009) Infection with Rhabdias tokyoensis (Nematoda: Rhabdiasidae) in European captive-bred swordtail newts, Cynops ensicauda (Amphibia: Salamandridae). Salamandra 45(2):91-94

Fernández Loras A, Hidalgo-Vila J, Hermosilla C, García G, López J, Duffus ALJ, Cunningham AA, Roca V (2011) Preliminary health screening and possible pathogen determination in a Bufo bufo (Linnaeus, 1758) (Amphibia:Bufonidae) population. J Nat History 45:1-14

Foden WB, Butchart SHM, Stuart SN, Vié JC, Akçakaya HR, Angulo A, De Vantier LM, Gutsche A, Turak E, Cao L, Donner SD, Katariya V, Bernard R, Holland RA, Hughes AF, O'Hanlon SE, Garnett ST, Sekercioglu CH, Mace GM (2013) Identifying the world's most climate change vulnerable species: a systematic trait-based assessment of all birds, amphibians and corals. PLoS One 8:e65427

Frank W (1981) Endoparasites. In: Cooper JE, Jackson OF (eds) Diseases of the Reptilia, vol 1. Academic press, London, New York, Toronto, Sydney, San Francisco, pp 291-350

Frank W (1984) Infektion (Invasionen) mit Parasiten (Parasitosen). In: Isenbügel E, Frank W (eds) Heimtierkrankheiten. Ulmer, Stuttgart, pp 226-320
Frank W (1985) Non-hemoparasitic protozoans. In: Hoff GL, Frye FL, Jacobson ER (eds) Diseases of amphibians and reptiles. Plenum Press, New York and London, pp 260-384

Garner TWJ, Stephen I, Wombwell E, Fisher MC (2009) The amphibian trade: bans or best practice? Ecohealth 6:148-151. https://doi.org/ 10.1007/s10393-009-0233-1

Graczyk TK, Fried B (1998) Echinostomiasis: a common but forgotten food-borne disease. Am J Trop Med Hyg 58(4):501-504

Hallinger MJ, Taubert A, Hermosilla C, Mutschmann F (2018) Occurrence of health- compromising protozoan and helminth infections in tortoises kept as pet animals in Germany. Parasit Vector 11(1):352

Hallinger MJ, Taubert A, Hermosilla C, Mutschmann F (2019) Captive Agamid lizards in Germany: Prevalence, pathogenicity and therapy of gastrointestinal protozoan and helminth infections. Comparative immunology, microbiology and infectious diseases, 63,74-80

Hallinger MJ, Taubert A, Hermosilla C (2020) Occurrence of Kalicephalus, Strongyloides, and Rhabdias nematodes as most common gastrointestinal parasites in captive snakes of German households and zoological gardens. Parasitology Research, 119(3):947956

Hamann MI, Kehr AI, González CE (2012) Community structure of helminth parasites of Leptodactylus bufonius (Anura: Leptodactylidae) from northeastern Argentina. Zool Stud 51(8): 1454-1463

Heine J (1982) Eine einfache Nachweismethode für Kryptosporidien im Kot. Zbl Vet Med B 29:324-327

Hendrikx WML (1983) Observations on the routes of infection of Oswaldocruzia filiformis (Nematoda: Trichostrongylidae) in amphibia. Ze Parasitenkd 69:119-126

Henle K, Mester B, Lengyel S, Puky M (2012) A review of a rare type of anomaly in amphibians, tail duplication and bifurcation, with description of three new cases in European species (Triturus dobrogicus, Triturus carnifex, and Hyla arborea). J Herp 46:451455

Hill RL, Mendelson JR, Stabile JL (2015) Direct observation and review of herbivory in Sirenidae (Amphibia: Caudata). Southeast Nat 14(1): N5-N9

Hofrichter R (2000) The encyclopedia of amphibians: the world of frogs, toads, salamanders and newts. Firefly Books ISBN-10: $155209541 \mathrm{X}$

Jirků M, Valigurová A, Koudela B, Křížek J, Modrý D, Šlapeta J (2008) New species of Cryptosporidium Tyzzer, 1907 (Apicomplexa) from amphibian host: morphology, biology and phylogeny. Folia Parasitol (Praha) 55:81-94. https://doi.org/10.14411/fp.2008.011

Kehr AI, Hamann MI (2003) Ecological aspects of parasitism in the tadpole of Pseudis paradoxa from Argentina. Herpetol Rev 34(4): 336-341

Kia GS, Ukuma BI (2017) Cryptosporidium in wild frogs (Rana spp) consumed by humans in Kaduna State Nigeria. Online JPublic Health Inform 9(1)

Kia GS, Ukuma BU, Odoba MB, Okpanachi JU (2017) Occurrence of Cryptosporidium oocysts in edible frogs (Rana species) sold for human consumption in Hanwa Frog Market Zaria, Kaduna State, Nigeria. J Coast Life Med 5(5):202-205

Krautwald-Junghanns (2017) Haltung exotischer Tiere und Wildtiere in Privathand: Situationsanalyse, Bewertung und Handlungsbedarf insbesondere unter Tierschutzaspekten. 2. Zwischenbericht. Teil 3: Ergebnisse der Situationsanalyse Haltungsbedingungen/ Tierschutzprobleme übergeordnete Diskussion und Zusammenfassung Gegenüberstellung der geplanten zu den erreichten Zielen Literaturverzeichnis. https://service.ble.de/ptdb/ index 2.php?detail_id=57204\&site_key $=141 \&$ stichw $=$ 2815HS014\&zeilenzahl zaehler $=1 \#$ newContent. Accessed 26 Feb 2020 
Kuzmin Y, Tkach VV, Snyder SD (2001) Rhabdias ambystomae sp. n.(Nematoda: Rhabdiasidae) fröm the North American spotted salamander Ambystoma maculatum (Amphibia: Ambystomatidae). Comp Parasitol 68(2):228-235

Kuzmin Y, Tkach VV, Snyder SD (2003) The nematode genus Rhabdias (Nematoda: Rhabdiasidae) from amphibians and reptiles of the Nearctic. Comp Parasitol 70:101-114. https://doi.org/10.1654/4075

Langford GJ (2010) Ecology and relationships of Rhabdias Spp. (Nematoda: Rhabdiasidae) from North American amphibians and reptiles. Dissertation, University of Nebraska at Lincoln

Langford GJ, Janovy J Jr (2009) Comparative life cycles and life histories of North American Rhabdias spp. (Nematoda: Rhabdiasidae): lungworms from snakes and anurans. J Parasitol 95:1145-1155. https:// doi.org/10.1645/ge-2044.1

Loras AL, Hidalgo-Vila J, Hermosilla C, García G, López J, Duffus ALJ, Cunningham AA, Roca V (2010) Preliminary health screening and possible pathogen determination in a Bufo bufo (Linneaus, 1758) (Amphibia: Bufonidae) population. J Nat Hist 45(1-2):1-14

Marcogliese DJ, Pietrock M (2011) Combined effects of parasites and contaminants on animal health: parasites do matter. Trends Parasitol 27:123-130. https://doi.org/10.1016/j.pt.2010.11.002

Marenzoni ML, Zicavo A, Veronesi F, Morganti G, Scuota S, Coletti M, Passamonti F, Santoni L, Natali M, Moretta I (2015) Microbiological and parasitological investigation on chelonians reared in Italian facilities. Vet Ital 51:173-178. https://doi.org/10. 12834/VetIt.7.21.3

Martel A, Blooi M, Adriaensen C, Van Rooij P, Beukema W, Fisher MC, Farrer RA, Schmidt BR, Tobler U, Goka K, Lips KR, Muletz C, Zamudio KR, Bosch J, Lötters S, Wombwell E, Garner TWJ, Cunningham AA, Spitzen-van der Sluijs A, Salvidio S, Ducatelle R, Nishikawa K, Nguyen TT, Kolby JE, Van Bocxlaer I, Bossuyt F, Pasmans F (2014) Recent introduction of a chytrid fungus endangers Western Palearctic salamanders. Science 346:630-631

Mohammad MK, Al-Moussawi AA, Jasim SY (2010) Helminth parasites of the green toad Bufo viridis Laurenti, 1768 in Baghdad Area, Central Iraq. Egypt Acad J Biol Sci 2(1):17-25

Mutschmann F (2010) Erkrankungen der Amphibien. 2., überarbeitete und erweiterte Auflage. Enke Verlag, Stuttgart, pp 235-284

Nguyen TT, Nguyen TV, Ziegler T, Pasmans F, Martel A (2017) Trade in wild anurans vectors the urodelan pathogen Batrachochytrium salamandrivorans into Europe. Amphibia-Reptilia 38:554-556

Pantchev N, Tappe D (2011) Pentastomiasis and other parasitic zoonoses from reptiles and amphibians. Berl Muench Tieraerztl Wochenschr 124(11-12):528-535

Patterson-Kane JC, Eckerlin RP, Lyons ET, Jewell MA (2001) Strongyloidiasis in a Cope's grey tree frog (Hyla chrysoscelis). J Zoo Wildl Med 32:106-110. https://doi.org/10.1638/10427260(2001)032[0106:SIACSG]2.0.CO;2
Pasmans F, Blahak S, Martel A, Pantchev N (2008) Introducing reptiles into a captive collection: the role of the veterinarian. The Veterinary Journal, 175(1); 53-68

Rizvi AN, Bhutia PT (2010) Helminth parasite communities in anuran amphibians of Kalesar Wildlife Sanctuary (Haryana). India J Parasit Dis 34:79-82. https://doi.org/10.1007/s12639-010-0016-1

Rizvi AN, Bursey CR, Bhutia PT (2011) Cosmocerca kalesari sp. nov.(Nematoda, Cosmocercidae) in Euphlyctis cyanophlyctis (Amphibia, Anura) from Kalesar Wildlife Sanctuary, Haryana, India. Acta Parasitologica, 56(2):202-207

Stuart SN, Chanson JS, Cox NA, Young BE, Rodrigues AS, Fischman DL, Waller RW (2004) Status and trends of amphibian declines and extinctions worldwide. Science 306(5702):1783-1786

Thiemann GW, Wassersug RJ (2000) Patterns and consequences of behavioural responses to predators and parasites in Rana tadpoles. Biol J Linn Soc 71:513-528

Vaucher C (1990) Polystoma cuvieri n. sp. (Monogenea: Polystomatidae), a parasite of the urinary bladder of the leptodactylid frog Physalaemus cuvieri in Paraguay. J Parasitol 76: 501-504. https://doi.org/10.2307/3282828

Warwick C, Arena PC, Steedman C (2012) Visitor behaviour and public health implications associated with exotic pet markets: an observational study. JRSM Short Rep 3:63

Wren S, Angulo A, Meredith H, Kielgast J, Dos Santos M, Bishop P (2015) Amphibian conservation action plan. April 2015. IUCN SSC Amphibian Specialist Group

Wolf D, Vrhovec MG, Failing K, Rossier C, Hermosilla C, Pantchev N (2014) Diagnosis of gastrointestinal parasites in reptiles: comparison of two coprological methods. Acta Veterinaria Scandinavica, 56(1), 44

Yildirimhan HS, Sümer N, İncedoğan S, Bursey CR (2012) Helminth parasites of the lemon-yellow tree frog, Hyla savignyi (Hylidae), from Turkey. Turk J Zoo 36(2):171-184. https://doi.org/10.3906/ zoo-1006-9

Yoshikawa H, Abe N, Wu Z (2004) PCR-based identification of zoonotic isolates of Blastocystis from mammals and birds. Microbiology 150(5):1147-1151

Ziegler T (2016) Two decades of herpetodiversity research in Vietnam and Laos: a review of a German-Vietnamese long-term cooperation. Proceedings of the 3rd National Scientific Conference on Amphibians and Reptiles in Vietnam, Hanoi, 26 November 2016

Ziegler T; Rauhaus A (2019) Der Beitrag des Kölner Zoos zur Erhaltung der Amphibienvielfalt: Nachzucht-, Forschungs- und Schutzprojekte. Zeitschrift des Kölner Zoos, Heft 2/2019. 62. Jahrgang

Publisher's note Springer Nature remains neutral with regard to jurisdictional claims in published maps and institutional affiliations. 